\title{
Intracardiac device and prosthetic infections: What do we know?
}

\author{
B Lynn Johnston $M D^{1}$, John $M$ Conly $M D^{2}$
}

$\mathrm{I}_{\mathrm{ota}}^{\mathrm{n}}$

nfection of an intracardiac device or prosthesis represents one of the more common reasons for infectious diseases consultation in many hospital-based practices. As more patients receive pacemakers or implanted defibrillators and undergo valve replacement, it stands to reason that we will see more patients with infections involving these devices. The management of these challenging infections requires knowledge of both the medical and surgical literature and a multidisciplinary approach involving cardiologists, cardiothoracic surgeons, infectious diseases physicians and microbiologists.

Several prospective and retrospective studies have examined the rate of prosthetic valve endocarditis (PVE). PVE is divided into early and late, according to the time since surgery. Cases falling within the first two months of surgery are considered to be early, although some authors consider early to be all cases occurring within the first 12 months, because this also represents the time frame for a surgical site infection in the presence of an implant. Others consider this two to 12 month period to be intermediate. Cases occurring outside the above time frames are late PVE. In examining rates and etiologies in the literature, these differences in definitions must be considered.

Using 12 months as the cut-off, Gordon et al (1) determined that $1 \%$ of patients undergoing valve surgery at the Cleveland Clinic between 1992 and 1997 developed early PVE. The risk decreased from $1.5 \%$ to $0.7 \%(\mathrm{P}<0.01)$ from 1992 to 1997 . This is similar to the $0.94 \%$ rate reported in a German cohort where early PVE was considered to occur within 60 days (2). These authors also noted a significant decline in risk for early PVE over time, from before 1975 to 1992 (2). In a Canadian study (3), 1.5\% of patients developed late (after 60 days) PVE. The yearly risk of developing PVE after the first 12 months is estimated to be between $0.3 \%$ and $0.6 \%$ $(2,4)$ with the five-year cumulative risk reported as $3.0 \%$ to $5.7 \%$ (4). There is little recently published data comparing the PVE risk associated with either valve type (bioprosthesis or mechanical) or site (aortic versus mitral), and the available data are conflicting. Although there is a suggestion that bioprosthetic valves and valves at the aortic position carry a slightly higher risk, the differences are small and not likely to be clinically relevant $(1,2,4)$.

The etiology of PVE is well described. Although there is a broad range of potential pathogens, most cases are due to a relatively small number of organisms with some distinction between early and late PVE. Staphylococcus aureus and coagulasenegative are the predominant organisms in early PVE, regardless of a definition of less than two or less than 12 months $(1,4-8)$. The predominant organisms in late PVE are, not surprisingly, similar to those causing native valve endocarditis (NVE) with streptococci, enterococci and $\mathrm{S}$ aureus most commonly isolated $(2,4,5,7,9)$. Gram-negative bacilli, fungi and fastidious Gramnegative coccobacilli belonging to the Haemophilus species, Actinobacillus actinomycetemcomitans, Cardiobacterium hominis, Eikenella corrodens, and Kingella species (HACEK) group are more unusual causes (10\% or less) with Gram-negative bacilli and fungi tending to occur with early PVE, and HACEK organisms with late PVE (4-6). Approximately 3\% to $10 \%$ of PVE is culture-negative $(2,4,5,9)$.

The clinical features of PVE have been well described. Investigators in Israel reviewed 108 cases of endocarditis diagnosed during the years 1990 to 1999 and found no clinical features that distinguished native and prosthetic valve endocarditis (10), in keeping with prior impressions $(4,11)$. In particular, embolic findings occur no more nor less frequently $(10,12,13)$. Others have reported somewhat different findings. One group (14) found that patients with PVE more commonly had atrial fibrillation and sepsis, and another (8) found that they more commonly had congestive heart failure and sepsis and less frequently had vegetations. In one study (15), the Duke criteria were found to be $79 \%$ sensitive in making a definite diagnosis of PVE in 76 surgically confirmed cases. The sensitivity of the Duke criteria could be increased to $97 \%$ for late PVE by adding heart failure and conduction disturbances to the minor criteria. The specificity of the criteria could not be assessed.

Treatment guidelines for staphylococcal PVE generally recommend a combination of two or three agents, regardless of species or methicillin susceptibility $(4,16,17)$. Rifampin is suggested because of its excellent tissue penetration, ability to enter phagocytes and kill intracellular organisms and ability to kill stationary organisms (18). However, rifampin resistance can develop, even in the setting of combination therapy (19-22). Because of its relatively high intrinsic resistance rate, rifampin resistance is more likely to develop in the presence of a large number of organisms. It has therefore been suggested,

\footnotetext{
${ }^{1}$ Queen Elizabeth II Health Sciences Centre and Dalhousie University, Halifax, Nova Scotia; ${ }^{2}$ Departments of Pathology and Laboratory

Medicine, Medicine, and Microbiology and Infectious Diseases, University of Calgary, Calgary, Alberta

Correspondence and reprints: Dr B Lynn Johnston, Queen Elizabeth II Health Sciences Centre, 1270 Tower Road, Halifax, Nova Scotia B3H 2 Y9.

Telephone 902-978-8229, fax 902-473-7394, e-mail ljohnsto@dal.ca

Received and accepted for publication June 21, 2004
} 
that to prevent resistance, regimens selected for treatment of staphylococcal PVE should contain two active antistaphylococcal antibiotics (4). Where this cannot be done, it has been suggested that rifampin should be started three to five days after a single agent has been administered (4).

The evidence from in vitro and in vivo animal studies that examine the interactions between various combinations of antibacterials against susceptible and resistant strains of staphylococci is less than robust. The in vitro studies consist primarily of time-kill curves that look at rates of bacterial killing for combinations of antibiotics compared with the single agents and determine whether the combination is synergistic, antagonistic or indifferent. Results are quite consistent for rifampin, with indifference or antagonism demonstrated when it is used in combination with nafcillin, vancomycin or teicoplanin against methicillin-susceptible $S$ aureus (MSSA) (20); antagonism, indifference or synergy versus MSSA when combined with oxacillin (23); no evidence for synergy when combined with cephalothin, nafcillin, gentamicin or vancomycin for methicillin-resistant Staphylococcus epidermidis (MRSE) (24); and antagonism when added to vancomycin and gentamicin against MRSE and indifference when added to vancomycin alone (25). Thus, in vitro data provide no evidence to support a benefit of combination therapy. However, it can be argued that in vitro studies bear no resemblance to the clinical situation and extrapolations regarding efficacy should not be made from such information.

To explore the potential benefit of combination therapy in treating endocarditis, including that involving devices, a number of in vivo studies using the rabbit endocarditis model have been performed. In these experiments, a transaortic valve catheter is left in place throughout the experiment to simulate the device situation. The animals are then infected intravascularly with the test organism. Treatment is initiated one to six days after infection and continued for two to seven days, depending on the experimental design. Blood and valve cultures are obtained and the animals sacrificed at different time intervals. In a model of MSSA endocarditis, only very high doses of rifampin $(20 \mathrm{mg} / \mathrm{kg})$ added to cloxacillin reduced vegetation titres more than cloxacillin alone (26). Overall, the addition of rifampin was beneficial in three of five regimens tested, did not affect the rate of sterilization in one and reduced the effectiveness of the beta-lactam in the other (26). As expected, Archer et al (24) found that cephalothin was ineffective in MRSE endocarditis, whereas rifampin was effective against rifampin susceptible strains. Another group (27) found no survival differences among rabbits with MRSE endocarditis treated with different single agent regimens. Not surprisingly, there were fewer organisms per valve and a greater proportion of sterile valves in rabbits treated with vancomycin, gentamicin or rifampin as compared with a beta-lactam in these experiments, with no combinations tested (27). Finally, in a set of experiments published in 1983, the combination of vancomycin, gentamicin and rifampin was more effective than any of the agents used singly or in combination in sterilizing vegetations associated with MRSE endocarditis (25). In experimental foreign body infection caused by methicillin-resistant $S$ aureus, Swiss researchers found that a combination of rifampin with vancomycin or fleroxacin (an early fluoroquinolone) was more effective than either of the latter two drugs alone (21). Thus, for methicillin-resistant strains, the experimental evidence suggests a benefit to combination therapy, but does not point to the optimal combination of agents. There is insufficient data for MSSA to conclude a potential benefit.

There is very little literature examining the outcomes of various regimens in the treatment of human staphylococcal PVE. In 1983, Karchmer et al (28) described their experience with 23 patients with MRSE PVE. Patients received rifampin $900 \mathrm{mg}$ to $1200 \mathrm{mg}$ daily in combination with vancomycin or a beta-lactam. The vancomycin combination was more effective than the beta-lactam one ( $87 \%$ versus $38 \%, \mathrm{P}=0.025)$. The numbers were too small to compare outcomes in patients receiving combination therapy (rifampin \pm gentamicin) versus vancomycin alone. That same year, these authors published (29) their experience with 70 patients having 75 episodes of S epidermidis PVE. Of 68 episodes analyzed for outcome, analysis of antibiotic efficacy was limited to 46 episodes treated for more than six days and for which the causative organism was confirmed as being methicillin-resistant. Patients were categorized as receiving rifampin ( $900 \mathrm{mg}$ to $1200 \mathrm{mg}$ once daily) or an aminoglycoside if they had it for at least 10 days. Fifty-two per cent of patients received rifampin \pm aminoglycoside. Again, a vancomycin regimen was better than a beta-lactam one $(81 \%$ versus $50 \%$ cure, $\mathrm{P}=0.055)$. There was a trend to more cures when rifampin \pm aminoglycoside were used in combination with vancomycin, compared with vancomycin alone (90\% versus $50 \%, \mathrm{P}=0.06$ ).

Risk factors for death were reviewed in 33 patients diagnosed with S aureus PVE between January 1975 and January 1983 (30). The majority (73\%) of patients received an aminoglycoside in addition to a beta-lactam or vancomycin but only $33 \%$ also received rifampin. No comparisons of outcome according to antimicrobial regimen were made. It may be that rifampin use has improved in the last 20 years. However, this study suggests that its use, despite guidelines, is limited.

A more recent study in New Zealand (18) looked at outcomes of patients undergoing valve replacement for endocarditis from September 1963 through December 1999. Sixty-one patients had staphylococcal PVE, 29 due to MSSA and 32 due to coagulase-negative staphylococci (56\% MRSE). Analyses did not distinguish outcomes for methicillin-resistant versus -sensitive strains. Most patients received dual therapy with either rifampin or an aminoglycoside in addition to vancomycin or a beta-lactam. Only six patients (10\%) received triple therapy for all or part of their treatment course. After adjusting for duration of therapy, valves from patients receiving any kind of combination (two to three drugs for all or part of the treatment course) were five to nine times more likely to be culture-negative than those receiving monotherapy $(\mathrm{P}=0.024)$. The rate of valve sterilization was similar with and without rifampin (67\% versus $63 \%$ ). Thus, while this study supports combination therapy in the management of PVE, it does not clarify the optimal combination or whether it applies equally to methicillin-susceptible and-resistant strains. Eightythree per cent of patients treated more than 14 days were culture-negative at the time of surgery.

The overall mortality for PVE has been reportedto be as high as $50 \%$, with early PVE having a higher mortality than late $(2,3,7)$. Whether it carries a poorer outcome than NVE is not clear. In a study comparing aortic native and prosthetic valve endocarditis, PVE carried a higher operative mortality, but long-term survival for discharged patients was similar at five years $(81 \pm 3 \%)$ and 10 years $(63 \pm 5 \%)(8)$. On the other 
hand, PVE is an independent predictor of early and late mortality in patients with mitral valve endocarditis (14). In a longterm follow-up study of patients with endocarditis, PVE was not independently associated with a higher death rate, but was a predictor of the need for valve replacement compared with patients with NVE (31). Infection with a nonstreptococcal organism, particularly $S$ aureus, is associated with a poorer outcome in patients with PVE $(6,32,33)$. Most studies have shown a survival benefit for patients treated both surgically and medically for early and late PVE $(1,3,6,7)$. One caveat is that patients not having surgery may represent patients with greater burden of illness and inherently poorer outcome. This benefit appears most pronounced for patients with $S$ aureus PVE $(1,6,7,30)$, with no difference in surgical versus medical therapy for patients with PVE due to other organisms (6). Relapse of PVE or subsequent cardiac reoperation was more common in patients receiving medical treatment alone (5). Thus, a number of medically treated patients will eventually go on to valve surgery $(6,31-33)$. Other independent predictors of poor outcome include age $(3,31,34)$, recurrent endocarditis (31), severity of illness or other comorbidity $(3,6,7)$, cardiac complications $(5,6,8,30)$ and emergency surgery $(34)$.

One of the concerns related to surgical intervention in patients with PVE is the risk of relapsed or recurrent infection. Aranki et al $(8,14)$ looked at valve replacement in relationship to the course of antimicrobial therapy. Endocarditis was labelled 'active' if the patient required surgery before completing a standard course of antibiotics, regardless of whether cultures were positive at the time of surgery. For aortic valve endocarditis, the active group had higher operative mortality, greater recurrence rate, but no mortality difference, compared with a healed endocarditis group (8). There were no differences observed with mitral endocarditis (14). Using a similar definition, Guerra et al (34) identified 141 patients with PVE and NVE who required surgical intervention in the setting of active infection. Operative mortality was 30.5\%, and not significantly higher for PVE compared with NVE. Excluding operative mortality, survival was $87 \%$ at five years and $84 \%$ at 10 years, again with no difference for PVE. Survival free from infection was $93 \%$ at five years and $83 \%$ at 10 years. In another study (35), the mortality in 11 patients with PVE who had surgery within $48 \mathrm{~h}$ of a positive blood culture or evidence of active at the time of operation was $9 \%$. One patient required repeat surgery nine months later for valve dehiscence, but the explanted valve was culture-negative (35). Wolf et al (6) found that having positive valve cultures or eight or less days of antibiotics before surgery were not predictive of poor outcome. Two studies $(36,37)$ of valve replacement for complicated endocarditis (one study had both PVE and NVE, all with paravalvular abscess; the other was PVE with abscesses in $81 \%$ ) had operative mortality ranging from $9 \%$ to $13 \%$ and five- and eight-year freedom from recurrent endocarditis rates of $96 \%$ and $76 \%$, respectively. These studies point to a reinfection or relapse rate lower than generally perceived, even in the presence of active infection at the time of surgery.

Another major concern with PVE is the risk for central nervous system (CNS) complications, particularly if surgery is being contemplated. Neurological complications are reported in $6 \%$ to $28 \%$ of patients with PVE $(6,10,29,38,39)$. Embolic events are more likely to occur within two weeks of starting therapy $(12,30,33,38)$. The role of anticoagulation in cerebrovascular accidents was examined in 63 patients with PVE hospitalized between May 1972 and November 1987 in France (40). Seven had a cerebrovascular accident (five infarcts; two hemorrhages) with no deaths attributed to stroke and no difference in risk related to anticoagulation. In an earlier study (41), a Mayo Clinic cohort was reviewed for CNS complications associated with PVE. Patients with and without adequate anticoagulation (all had mechanical valves) were compared. Patients with adequate anticoagulation were less likely to have CNS complications than those where anticoagulation was suboptimal ( $8 \%$ versus $71 \%, \mathrm{P}<0.001$ ) although mortality was the same, indicating that anticoagulation did not adversely affect mortality or CNS morbidity. In a review of 50 patients with PVE in France (9), the authors also found a higher risk of CNS complications in inadequately anticoagulated patients and that heparin offered better control of anticoagulation than warfarin

Undertaking cardiopulmonary bypass in the setting of a CNS complication raises concern among physicians, although studies have generally not examined this as an independent predictor of outcome. A retrospective study (39) of 40 patients with cerebral complications of PVE found an operative mortality rate of $33.3 \%$, much higher than that for NVE, but no higher than the overall operative mortality for all patients with PVE. Looking at all patients with endocarditis (PVE and NVE), operative mortality was highest within $24 \mathrm{~h}$ after the onset of cerebral infarction $(66.3 \%), 10 \%$ to $31.3 \%$ from two days to four weeks, and $7 \%$ more than four weeks after infarct. The risk of cerebral complication decreased from $45.5 \%$ within $24 \mathrm{~h}$, to $10 \%$ for operations within 15 to 21 days, and reached $2.3 \%$ for those operated on more than four weeks later. These findings suggest that if surgery can be delayed following a CNS event, patients do better.

There is considerably less literature on infections associated with other intracardiac devices. Implantable cardioverter defibrillators (ICDs) were introduced into clinical practice in 1980 and the number inserted has increased over time (42). The ICD is similar in appearance to a pacemaker, but is larger (43). Early systems were placed via sternotomy or thoracotomy, with transvenous approaches most commonly performed since the 1990s (42). Not surprisingly, the number of infections associated with these devices has also increased, although the infection rate itself has decreased with improvements in surgical technique and transvenous insertion (4). Similarly to PVE, infections associated with these devices are divided into early (less than one month) and late, although in some studies, the terms late (one to 12 months) and delayed (more than 12 months) are used (4). Infection can involve the defibrillator pocket, electrodes, mesh patches in the subcutaneous tissues or on the heart surface, or endocardium. Most infections involve the pocket (44). The infection rate after ICD insertion ranges from $1 \%$ to $12 \%(42-47)$. Most present within one year of implantation (42-44). Rates are lowest for ICDs implanted via transverse approach (47). One study (43) found that the only risk for infection was being diabetic, with no relationship to age, sex or duration of surgery. The etiological agents mirror those of PVE, with $S$ aureus and coagulase-negative organisim most commonly isolated $(42-44,48)$. Blood cultures are positive in approximately $33 \%$ (48) and radiographic imaging may be difficult to interpret $(42,44)$.

Device explantation is considered to be the optimal management for ICD infection $(42-44,47,48)$. It has been noted to be safe and effective for ICDs placed through the 
transvenous approach (47). However, some have argued that this is not always necessary and in certain situations, it cannot be safely or easily accomplished $(42,44)$, particularly where there are epicardial leads and/or patches. In some instances, apparent cure has been accomplished by partial removal of the implant, usually the generator $(42,44)$. However, one group found that attempts to salvage the device by partial removal and antimicrobials failed in five of seven infections (46). Three of six patients in another study who did not have complete removal relapsed (48). There are no data describing the optimal regimen or duration of antimicrobial therapy. A review of 123 patients with ICD infection reported that patients received intravenous and/or oral antibiotics for a median of approximately three to 28 days (48). Overall mortality has been estimated at $8 \%$ to $12 \%$ $(44,48)$. Intracardiac devices and prostheses will continue to represent a source of infection for patients who receive them.

\section{REFERENCES}

1. Gordon SM, Serkey JM, Longworth DL, Lytle BW, Cosgrove III DM. Early onset prosthetic valve endocarditis: The Cleveland Clinic Experience 1992-1997. Ann Thorac Surg 2000;69:1388-92.

2. Horstkotte D, Piper C, Niehues R, Wiemer M, Schultheiss HP. Late prosthetic valve endocarditis. Eur Heart J 1995;16(Suppl B):39-47.

3. Sett SS, Hudon MPJ, Jamieson WRE, Chow AW. Prosthetic valve endocarditis. Experience with porcine bioprostheses. J Thorac Cardiovasc Surg 1993;105:428-34.

4. Karchmer AW, Longworth DL. Infections of intracardiac devices. Cardiol Clin 2003;21:253-71.

5. Calderwood SB, Swinski LA, Karchmer AW, Waternaux CM, Buckley MJ. Prosthetic valve endocarditis. Analysis of factors affecting outcome of therapy. J Thorac Cardiovasc Surg 1986;92:776-83.

6. Wolff M, Witchitz S, Chastang C, Régnier B, Vachon F. Prosthetic valve endocarditis in the ICU. Prognostic factors of overall survival in a series of 122 cases and consequences for treatment decision. Chest 1995;108:688-94.

7. Yu VL, Fang GD, Keys TF, et al. Prosthetic valve endocarditis: Superiority of surgical valve replacement versus medical therapy only. Ann Thorac Surg 1994;58:1073-7.

8. Aranki SF, Santini F, Adams DH, et al. Aortic valve endocarditis: Determinants of early survival and late morbidity. Circulation 1994;90:II175-82.

9. Leport C, Vilde JL, Bricaire F. Fifty cases of late prosthetic valve endocarditis: Improvement in prognosis over a 15 year period. Br Heart J 1987;58:66-71.

10. Fefer P, Raveh D, Rudensky B, Schlesinger Y, Yinnon AM. Changing epidemiology of infective endocarditis: A retrospective survey of 108 cases, 1990-1999. Eur J Clin Microbiol Infect Dis 2002;21:432-7.

11. Mullany CJ, Chua YL, Schaff HV, et al. Early and late survival after surgical treatment of culture-positive active endocarditis. Mayo Clin Proc 1995;70:517-25.

12. Vilacosta I, Graupner C, San Román JA, et al. Risk of embolization after institution of antibiotic therapy for infective endocarditis. J Am Coll Cardiol 2002;39:1489-95.

13. Durante Mangoni E, Adinolfi LE, Tripodi MF, et al. Risk factors for "major" embolic events in hospitalized patients with infective endocarditis. Am Heart J 2003;146:311-6.

14. Aranki SF, Adams DH, Rizzo RJ, et al. Determinants of early mortality and late survival in mitral valve endocarditis. Circulation 1995;92:II143-9.

15. Pérez-Vázquez A, Fariñas MC, Garcia-Palomo JD, et al. Evaluation of the Duke criteria in 93 episodes of prosthetic valve endocarditis: Could sensitivity be improved? Arch Intern Med 2000;160:1185-91.

16. Wilson WR, Karchmer AW, Dajani AS. Antibiotic treatment of adults with infective endocarditis due to streptococci, enterococci, staphylococci, and HACEK organisms. JAMA 1995;274:1706-13.
Optimal management requires consideration of antimicrobial, anticoagulation and surgical options. While the antibiotic treatment guidelines for staphylococcal PVE call for a combination of beta-lactam or vancomycin with gentamicin and rifampin, in practice this regimen is not consistently followed and, in fact, the optimal regimen is undefined. Patients with $S$ aureus PVE have improved outcomes if treated surgically and medically. Patients with complicated PVE also do better with combined surgical and medical management, compared with medical management alone. Required valve replacement should not be delayed to complete antibiotic therapy. Survival, free from infection, is satisfactory even when surgery is performed during active infection. In patients with mechanical valves, anticoagulation should be continued. Surgery is an essential component of management for ICD infections. The appropriate antimicrobial regimen for these infections is not well defined.

17. The Sanford Guide to Antimicrobial Therapy. In: Gilbert DN, Moellering RC Jr, Sande MA, Eliopoulos GM, eds. Antimicrobial Therapy Inc, 34th edn. Vermont: Hyde Park 2004:20.

18. Drinkovic D, Morris AJ, Pottumarthy S, MacCulloch D, West T. Bacteriological outcome of combination versus single-agent treatment for staphylococcal endocarditis. J Antimicrob Chemother 2003;52:820-5.

19. Hackbarth CJ, Chambers HF, Sande MA. Serum bactericidal activity of rifampin in combination with other antimicrobial agents against Staphylococcus aureus. Antimicrob Agents Chemother 1986;29:611-3.

20. Rouse MS, Wilcox RM, Henry NK, Steckelberg JM, Wilson WR Ciprofloxacin therapy of experimental endocarditis caused by methicillin-resistant Staphylococcus epidermidis. Antimicrob Agents Chemother 1990;34:273-6.

21. Lucet JC, Herrmann M, Rohner P, et al. Treatment of experimental foreign body infection caused by methicillinresistant Staphylococcus aureus. Antimicrob Agents Chemother 1990;34:2312-7.

22. Simon GL, Smith RH, Sande MA. Emergence of rifampinresistant strains of Staphylococcus aureus during combination therapy with vancomycin and rifampin: A report of two cases. Rev Infect Dis 1983;5:S507-8.

23. Van der Auwera P, Klastersky J. In vitro study of the combination of rifampin with oxacillin against Staphylococcus aureus. Rev Infect Dis 1983;5(Suppl 3):S509-13.

24. Archer GL, Johnston JL, Vazquez GJ, Haywood III HB. Efficacy of antibiotic combinations including rifampin against methicillinresistant Staphylococcus epidermidis: In vitro and in vivo studies. Rev Infect Dis 1983;5:S538-42.

25. Kobasa WD, Kaye KL, Shapiro T, Kaye D. Therapy for experimental endocarditis due to Staphylococcus epidermidis. Rev Infect Dis 1983;5:S533-7.

26. Zak O, Scheld WM, Sande MA. Rifampin in experimental endocarditis due to Staphylococcus aureus in rabbits. Rev Infect Dis 1983;5:S481-90

27. Vazquez GJ, Archer GL. Antibiotic therapy of experimental Staphylococcus epidermidis endocarditis. Antimicrob Agents Chemother 1980;17:280-5.

28. Karchmer AW, Archer GL, Dismukes WE. Rifampin treatment of prosthetic valve endocarditis due to Staphylococcus epidermidis. Rev Infect Dis 1983;5:S543-7.

29. Karchmer AW, Archer GL, Dismukes WE. Staphylococcus epidermidis causing prosthetic valve endocarditis: Microbiologic and clinical observations as guides to therapy. Ann Intern Med 1983;98:447-55.

30. John MDV, Hibberd PL, Karchmer AW, Sleeper LA, Calderwood SB. Staphylococcus aureus prosthetic valve endocarditis: Optimal management and risk factors for death. Clin Infect Dis 1998;26:1302-9.

31. Mansur AJ, Dal Bó CMR, Fukushima JT, et al. Relapses, recurrences, valve replacements, and mortality during the long-term follow-up after infective endocarditis. Am Heart J 2001;141:78-86. 
32. Tornos P, Sanz E, Permanyer-Miralda G, et al. Late prosthetic valve endocarditis: Immediate and long-term prognosis. Chest 1992;101;37-41.

33. Tornos P, Almirante B, Olona M, et al. Clinical outcome and long-term prognosis of late prosthetic valve endocarditis: A 20-year experience. Clin Infect Dis 1997;24:381-6.

34. Guerra JM, Tornos MP, Permanyer-Miralda G, et al. Long-term results of mechanical prostheses for treatment of active infective endocarditis. Heart 2001;86:63-8.

35. Peri M, Vuk F, Huski R, et al. Active infective endocarditis: Low mortality associated with early surgical treatment. Cardiovasc Surg 2000;8:208-13.

36. d'Udekem Y, David TE, Feindel CM, Armstrong S, Sun Z. Longterm results of operation for paravalvular abscess. Ann Thorac Surg 1996;62:48-53.

37. Dossche KM, Defauw JJ, Ernst SM, et al. Allograft aortic root replacement in prosthetic aortic valve endocarditis: A review of 32 patients. Ann Thorac Surg 1997;63:1644-9.

38. Davenport J, Hart RG. Prosthetic valve endocarditis 1976-1987. Antibiotics, anticoagulation, and stroke. Stroke 1990;21:993-9.

39. Eishi K, Kawazoe K, Kuriyama Y, et al. Surgical management of infective endocarditis associated with cerebral complications: Multi-center retrospective study in Japan. J Thorac Cardiovasc Surg 1995;110:1745-55.

40. Delahaye JP, Poncet P, Malquarti V, et al. Cerebrovascular accidents in infective endocarditis: Role of anticoagulation. Eur Heart J 1990;11:1074-8.
41. Wilson WR, Geraci JE, Danielson GK, et al. Anticoagulant therapy and central nervous system complication in patients with prosthetic valve endocarditis. Circulation 1978;57:1004-7.

42. Samuels LE, Samuels FL, Kaufman MS, Morris RJ, Brockman SK. Management of infected implantable cardiac defibrillators. Ann Thorac Surg 1997;64:1702-6.

43. Spinler SA, Nawarskas JJ, Foote EF, et al. Clinical presentation and analysis of risk factors for infectious complications of implantable cardioverter-defibrillators at a university medical center. Clin Infect Dis 1998;26:1111-6.

44. Spratt KA, Blumberg EA, Wood CA, Kutalek SP, Reboli AC. Infections of implantable cardioverter defibrillators: Approach to management. Clin Infect Dis 1993;17:679-85.

45. Bigger JT Jr. Coronary Artery Bypass Graft (CABG) Patch Trial investigators. Prophylactic use of implanted cardiac defibrillators in patients at high risk for ventricular arrhythmias after coronaryartery bypass graft surgery. N Engl J Med 1997;337:1569-75.

46. Lai KK, Fontecchio SA. Infections associated with implantable cardioverter defibrillators placed transvenously and via thoracotomies: Epidemiology, infection control, and management. Clin Infect Dis 1998;27:265-9.

47. Mela T, McGovern BA, Garan H, et al. Long-term infection rates associated with the pectoral versus abdominal approach to cardioverter-defibrillator implants. Am J Cardiol 2001;88:750-3.

48. Chua JD, Wilkoff BL, Lee I, et al. Diagnosis and management of infections involving implantable electrophysiologic cardiac devices. Ann Intern Med 2000;133:604-8. 


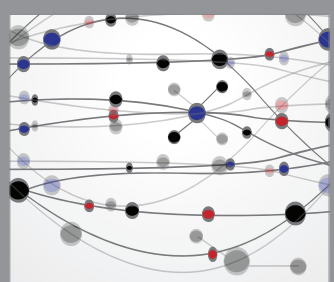

The Scientific World Journal
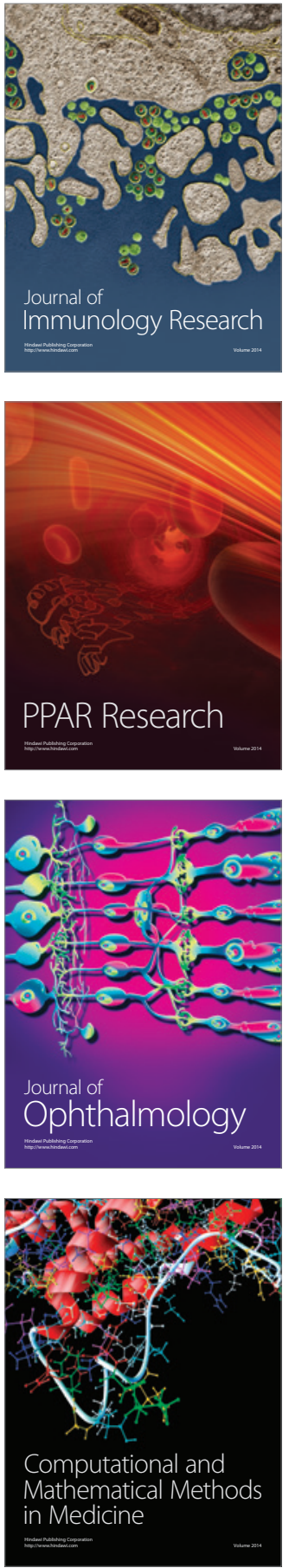

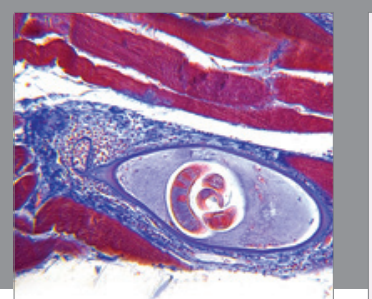

Gastroenterology Research and Practice

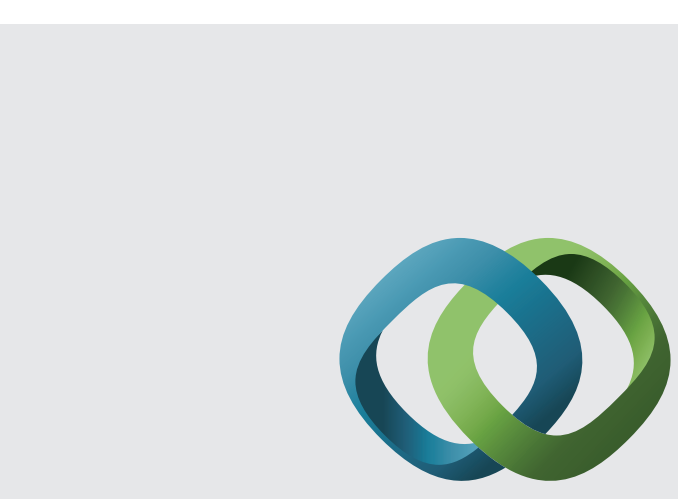

\section{Hindawi}

Submit your manuscripts at

http://www.hindawi.com
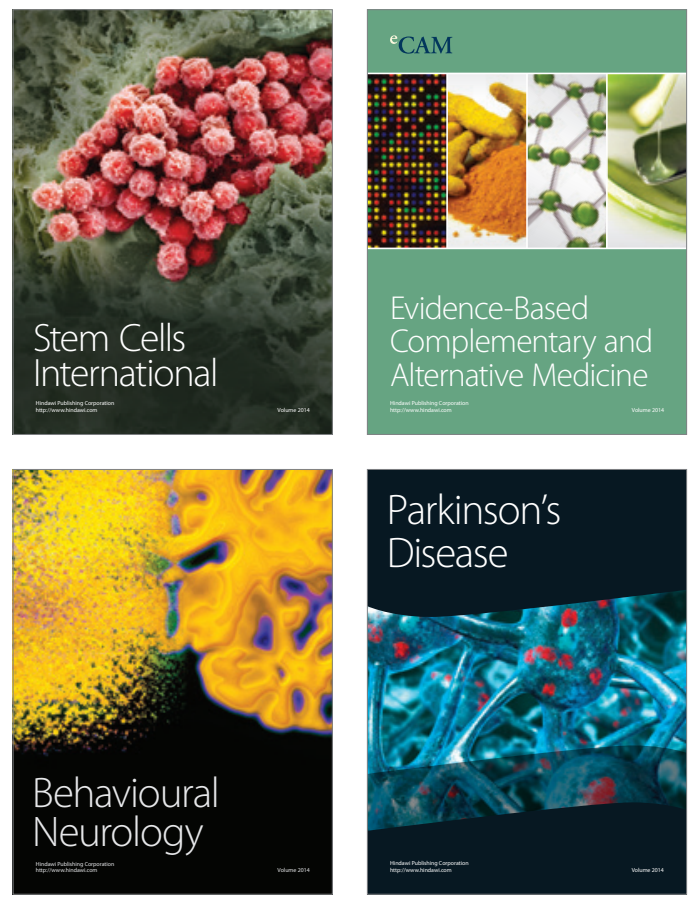
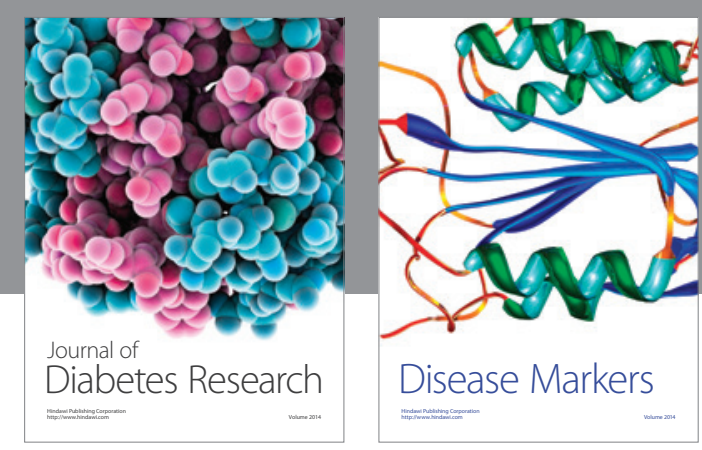

Disease Markers
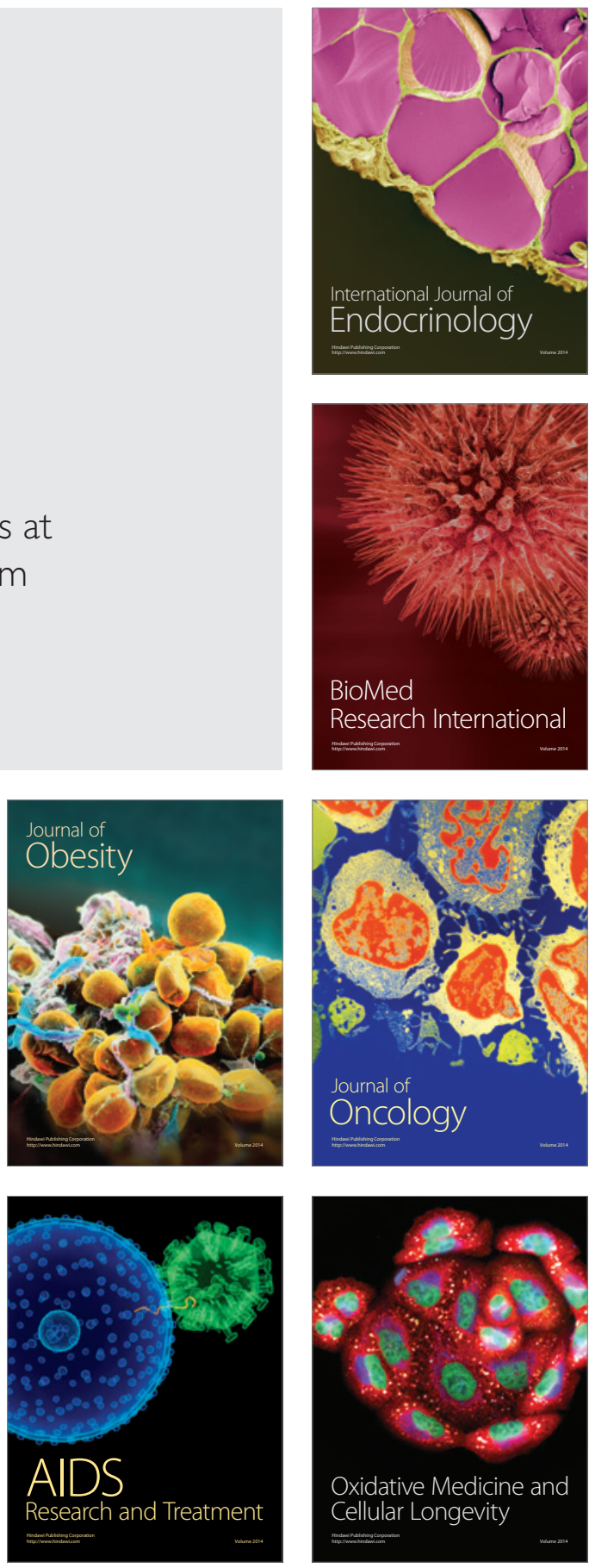